\title{
Cambios tecnológicos: interrogantes y desafíos del trabajo docente universitario virtualizado en Argentina
}

\author{
Technological changes: questions and challenges of virtualized \\ university teaching work in Argentina
}

\author{
Mara Mattioni \\ https://orcid.org/0000-0003-0266-0507 \\ maramattioni@hotmail.com \\ UNLaM | Argentina
}

\author{
Pablo Granovsky \\ https://orcid.org/0000-0003-2855-0963 \\ pgranovsky@yahoo.com.ar \\ UNLaM | Argentina
}

\section{RESUMEN}

La presente propuesta se enmarca en una investigación titulada “Cambios tecnológicos: nuevos desafíos para las relaciones laborales y la formación profesional" propuesta y desarrollada en el marco del Programa de Investigación Científica, Desarrollo y Transferencia de Tecnologías e Innovaciones (CyTMA 2) de la Universidad Nacional de La Matanza, cuya aspiración principal radica en caracterizar cómo los cambios tecnológicos afectan el campo de las relaciones laborales requiriendo de la formación de nuevas habilidades en los trabajadores.

En este sentido, como investigadores nos propusimos, al delinear el proyecto, enfocarnos sobre los actores del mundo del trabajo y las nuevas habilidades que deben adquirir como consecuencia de los cambios tecnológicos y las nuevas formas de empleo que se originan. Más específicamente, buscamos reconstruir la perspectiva de los actores del mundo del trabajo y del Estado asociados a las políticas de empleo y formación sobre el cambio tecnológico y organizacional en las relaciones laborales y en las competencias ocupacionales requeridas.

En Argentina, a partir de la aplicación del Aislamiento Social Preventivo y Obligatorio (ASPO) decretado por el gobierno nacional el 19 de marzo de 2020, con el objetivo de disminuir la tasa de contagio de la COVID-19, el mundo del trabajo se vio atravesado por un sinfín de modificaciones. Entre ellas, uno de las actividades mayormente atravesadas por los procesos de virtualización, resulto ser el trabajo docente evidenciando una notoria heterogeneización en cuanto a los alcances y posibilidades de los distintos sectores, niveles y casas de estudio en función de enfrentar las nuevas condiciones impuestas por la pandemia. Tensiones vinculadas a la "continuidad pedagógica" la "suspensión de clases presenciales" generaron debates y reflexiones en el ámbito universitario.

El propósito de la presente propuesta implica presentar un estado del arte respecto de los vectores que caracterizan al trabajo docente virtualizado actualmente en el ámbito de la Educación Superior, focalizando en las particularidades adquiridas en contexto del Aislamiento Social Preventivo y Obligatorio decretado por la Pandemia COVID 19. En este sentido, el objetivo de este trabajo es explorar las propuestas académicas divulgadas, e instalar la necesidad de la problematizar las diversas aristas de la virtualización de este trabajo de carácter presencial mayoritariamente en Argentina.

A nivel metodológico, para el desarrollo del proceso investigativo se emplearon fuentes secundarias. A nivel nacional se viabilizo la búsqueda a través de la colección COVID-19, un conglomerado de artículos sobre la temática que se encuentran alojados en los diversos repositorios de las instituciones que integran el Sistema Nacional de Repositorios Digitales (SNRD) y a nivel internacional en repositorios latinoamericanos (Red de repositorios Latinoamericanos) y españoles (Repositorio Digital de la Universidad de Barcelona).

\author{
PALABRAS CLAVE \\ trabajo docente, \\ cambios tecnológicos, \\ educación universitaria
}




\section{KEY WORDS}

teaching work, technological changes, university education

\section{ABSTRACT}

This proposal is part of an investigation entitled "Technological changes: new challenges for labor relations and professional training" proposed and developed within the framework of the Scientific Research, Development and Transfer of Technologies and Innovations Program (CyTMA 2) of the University Nacional de La Matanza, whose main aspiration lies in characterizing how technological changes affect the field of labor relations, requiring the training of new skills in workers.

In this sense, as researchers, we proposed, when outlining the project, to focus on the actors in the world of work and the new skills they should acquire as a consequence of the technological changes and the new forms of employment that originate. More specifically, we seek to reconstruct the perspective of the actors in the world of work and the State associated with employment and training policies on technological and organizational change in labor relations and in the required occupational skills.

In Argentina, from the application of the Preventive and Mandatory Social Isolation (ASPO) decreed by the national government on March 19, 2020, with the aim of reducing the contagion rate of COVID-19, the world of work was gone through endless modifications. Among them, one of the activities mainly crossed by the virtualization processes, turned out to be the teaching work evidencing a notorious heterogeneity regarding the scope and possibilities of the different sectors, levels and houses of study in order to face the new conditions imposed by the pandemic. Tensions linked to "pedagogical continuity" and the "suspension of face-to-face classes" generated debates and reflections at the university level. The purpose of this proposal involves presenting a state of the art regarding the vectors that characterize virtualized teaching work currently in the field of Higher Education, focusing on the particularities acquired in the context of Preventive and Mandatory Social Isolation decreed by the COVID Pandemic 19 In this sense, the objective of this work is to explore the academic proposals disclosed, and to install the need to problematize the various edges of the virtualization of this face-to-face work, mostly in Argentina. At the methodological level, secondary sources were used for the development of the investigative process. At the national level, the search was made possible through the COVID-19 collection, a conglomerate of articles on the subject that are housed in the various repositories of the institutions that make up the National System of Digital Repositories (SNRD) and at the international level in repositories Latin American (Network of Latin American repositories) and Spanish (Digital Repository of the University of Barcelona). 


\section{PRESENTACIÓN}

La presente propuesta se enmarca en una investigación titulada "Cambios tecnológicos: nuevos desafíos para las relaciones laborales y la formación profesional" propuesta y desarrollada en el marco del Programa de Investigación Científica, Desarrollo y Transferencia de Tecnologías e Innovaciones (CyTMA 2) de la Universidad Nacional de La Matanza, cuya aspiración principal radica en caracterizar cómo los cambios tecnológicos afectan el campo de las relaciones laborales requiriendo de la formación de nuevas habilidades en los trabajadores.

En este sentido, como investigadores nos propusimos, al delinear el proyecto, enfocarnos sobre los actores del mundo del trabajo y las nuevas habilidades que deben adquirir como consecuencia de los cambios tecnológicos y las nuevas formas de empleo que se originan. Más específicamente, buscamos reconstruir la perspectiva de los actores del mundo del trabajo y del Estado asociados a las políticas de empleo y formación sobre el cambio tecnológico y organizacional en las relaciones laborales y en las competencias ocupacionales requeridas.

como investigadores nos propusimos, al delinear el proyecto, enfocarnos sobre los actores del mundo del trabajo y las nuevas habilidades que deben adquirir como consecuencia de los cambios tecnológicos y las nuevas formas de empleo que se originan.

En Argentina, a partir de la aplicación del Aislamiento Social Preventivo y Obligatorio (ASPO) decretado por el gobierno nacional el 19 de marzo de 2020, con el objetivo de disminuir la tasa de contagio de la COVID-19, el mundo del trabajo se vio atravesado por un sinfín de modificaciones. Entre ellas, uno de las actividades mayormente atravesadas por los procesos de virtualización resulto ser el trabajo docente evidenciando una notoria heterogeneización en cuanto a los alcances y posibilidades de los distintos sectores, niveles y casas de estudio en función de enfrentar las nuevas condiciones impuestas por la pandemia. Tensiones vinculadas a la "continuidad pedagógica" y la "suspensión de clases pre- 
senciales" generaron debates y reflexiones en el ámbito universitario.

El propósito de la presente propuesta implica presentar un estado del arte respecto de los vectores que caracterizan al trabajo docente virtualizado actualmente en el ámbito de la Educación Superior, focalizando en las particularidades adquiridas en contexto del Aislamiento Social Preventivo y Obligatorio decretado por la Pandemia COVID 19. En este sentido, el objetivo de este trabajo es explorar las propuestas académicas divulgadas e instalar la necesidad de la problematizar las diversas aristas de la virtualización de este trabajo de carácter presencial mayoritariamente en Argentina.

A nivel metodológico, para el desarrollo del proceso investigativo se emplearon fuentes secundarias. A nivel nacional se viabilizo la búsqueda a través de la colección COVID-19, un conglomerado de artículos sobre la temática que se encuentran alojados en los diversos repositorios de las instituciones que integran el Sistema Nacional de Repositorios Digitales (SNRD) y a nivel internacional en repositorios latinoamericanos (Red de repositorios Latinoamericanos) y españoles (Repositorio Digital de la Universidad de Barcelona).

\section{DESARROLLO DE LA PROPUESTA}

A nivel nacional seleccionamos un artículo por demás sugerente y pertinente que forma parte de la Colección COVID-19 del Sistema Nacional de Repositorios Digitales denominado "El impacto asimétrico de la cuarentena. Estimaciones en base a una caracterización de ocupaciones".

La producción mencionada, escrita por dos investigadores de la Universidad Nacional de La Plata, focaliza en la relevancia del trabajo remoto y como este factor ha ido cobrando importancia en distintos sectores de actividad y empleo de un tiempo a esta parte. En este sentido refieren que "para conocer la posibilidad real del trabajo remoto para cada ocupación debemos indagar sobre sus características, actividades o atributos que nos permitan estimar en qué medida el teletrabajo es posible" (Foschiatti y Gasparini, 2020: 2), información que, desafortunadamente, no se encuentra disponible para nuestro país pero que apelando a la base de estudios de la O*NET es posible basar esta evaluación en dos dimisiones: las actividades que implica la ocupación y las características del entorno. En este sentido, la enseñanza conjuntamente con la Información y la comunicación; las actividades financieras y de seguros y las actividades profesionales, científicas y técnicas como una de las ramas de actividad con mayor posibilidad de mantener a sus trabajadores activos a través del teletrabajo (Foschiatti y Gasparini, 2020: 9).

Ante esta aparente factibilidad que el sector educativo parece connotar ante la virtualización de la tarea, es posible afirmar que resulta indiscutible que las herramientas sincrónicas (chats, videoconferencias en tiempo real) otorgan mayor posibilidad de interacción social y cognitiva atendiendo especialmente al componente tecnológico, la conectividad, la aplicación. Empero, también es necesario atender a 
que la implementación de prácticas educativas virtuales, el entretenimiento y el teletrabajo generan un tráfico en la red inesperado. En este sentido, los recursos y las habilidades necesarias de parte de los trabajadores docentes interpelan los modos de acceder a los mismos, el papel de las instituciones y del Estado principalmente ante la divergencia de condiciones dadas y posibles ante la heterogénea estructura social argentina (Foschiatti y Gasparini, 2020).

Observamos que cada planificación no logra ajustarse a los tiempos programados, las lecturas no logran hacerse con la profundidad que pretendemos y las explicaciones de los y las estudiantes redundan en la complejidad del tránsito de su cotidianeidad en este tiempo. Se expresan y muestran cansados de leer mediados por una pantalla, muchas veces no "los vemos" porque pausan el video "para garantizar mejor conectividad", otras nos encontramos con sus niños y niñas.

Por otro lado, abriendo el escenario a Latinoamérica, el artículo “Rol de los docentes ante la crisis del COVID-19. Una mirada desde el enfoque humano" escrito por docentes universitarios ecuatorianos se inclina por tensionar la subjetividad de los docentes y la necesidad de formación individual y colectiva de dichos trabajadores. A lo largo de la propuesta los autores enfatizan en la continuidad de las clases en su versión no presencial, destacando que los trabajadores docentes continúan en función con el impacto de nuevos estresores tales como la divergencia en la planificación, desarrollo y evaluación de las clases así como también la incidencia de los medios de comunicación y las redes sociales en los procesos de enseñanza aprendizaje (Villafuerte, Cevallos, y Vidal, 2020). Más allá del enfoque en las habilidades necesarias para la continuidad y/o inserción en este nuevo mundo del trabajo que contiene a los docentes universitarios, los autores destacan que "enseñar no es transferir conocimiento sino crear las posibilidades para su propia producción o construcción. Así, la labor docente se suma a los esfuerzos que han venido haciendo médicos, enfermeras, la policía y el ejército" (Villafuerte, Cevallos, y Vidal, 2020: 136). Así exploran los distintos procedimientos tales como el uso de la Tecnología de la Información y Comunicación (TIC) y la Tecnología para el Aprendizaje y Conocimiento (TAC) articulando pantallas, sonido y movimiento en procesos de enseñanza en función de volverlos más "atractivos" (Villafuerte, Cevallos, y Vidal, 2020). Esta recurrencia en la adjetivación que apuesta a vincular la enseñanza virtual con propuestas que atraigan o convoquen al alumnado pone sobre la mesa interrogantes al respecto de la concepción de trabajo docente, atendiendo a que pareciera que ante la virtualización las funciones se verían modificadas incluyendo aspectos lúdicos, dinámicos y atractivos. 
El artículo "Rol de los docentes ante la crisis del COVID-19. Una mirada desde el enfoque humano" enfatiza en el proceso que implica el pasaje de la presencialidad a la virtualidad a la hora de pensar en el trabajo docente; avanzando incluso sobre la cuestión de que esta contingencia pueda ser el punta pie para pensar en un cambio de concepción de la formación universitaria (Esteban Bara, 2020).

En esta línea, el autor se focaliza en tres beneficios de esta nueva forma de llevar adelante el trabajo docente:

...muchos profesores consideramos que esta nueva situación nos ha dado la oportunidad de realizar algo así como un curso avanzado en nuevas tecnologías (...); [también] hemos descubierto un auténtico mar de posibilidades formativas: (...) muchos profesores incorporaran la virtualidad en sus quehaceres docentes cuando todo esto pase y [por último] nos hemos dado cuenta de (...) lo necesario que es mejorar las condiciones socioeconómicas de no pocos estudiantes y profesores para que puedan cumplir con la formación universitaria dignamente. (Esteban Bara, 2020: 2)

Si bien el artículo focaliza en los beneficios a corto y mediano plazo para la educación superior en general a partir de este cambio basado en la tecnología; el docente de la Universidad de Barcelona menciona un riesgo relevante respecto de que la virtualización "podría desmontar la formación universitaria: una de las mejores producciones de la humanidad y acabar construyendo algo que ya no cumpla con su cometido" (Esteban Bara, 2020: 3). Esta arista del artículo dispara un sinfín de interrogantes secundarios a pensar la cuestión de la virtualización de los procesos de enseñanza aprendizaje eludiendo el análisis de este fenómeno en tensión con la categoría teórica del trabajo docente.

Adrián Almazán Gómez, docente de la Universidad Autónoma de Madrid, aboca el desarrollo del artículo "Covid-19: ¿Punto sin Retorno de la Digitalización de la Educación?" a cuestionar la posibilidad y la deseabilidad de la informatización de la educación apuntando a las condiciones de desigualdad tanto de trabajadores docentes como de estudiantes ante un escenario que requiere de tantas mediaciones ante la experiencia educativa aportando una mirada crítica al lo que proceso de informatización supone e implica (Gómez, 2020). Al respecto menciona "es indudable que la desigualdad social será inseparable de cualquier proceso de informatización que no se sustente sobre una fuerte inversión pública que garantice que los equipos y los conocimientos necesarios para su uso alcancen a todo tipo de familias por igual" (Gómez, 2020: 1). 
Al respecto de las particularidades del trabajo docente en el nivel universitario agrega que

...es imposible no ver que esta virtualización supone de facto la imposibilidad de vivenciar un elemento central en la formación universitaria: la formación de una personalidad crítica. Si pensamos que la Universidad debe ser algo más y algo diferente a un mero centro de formación profesional de alto nivel, entenderemos que el papel que juega la socialización cultural y política dentro y fuera del aula es fundamental. Y ésta, de nuevo, es imposible sin el encuentro con los otros. Ni un diálogo que vaya más allá del intercambio de monólogos ni la educación no formal que ofrece el contexto formativo amplio del espacio universitario es posible en una educación confinada. (Gómez, 2020: 2).

Sobre el final del artículo, el autor focaliza su análisis en el impacto concreto que los procesos de digitalización tienen en el trabajo docente proponiendo el desarrollo de códigos de buenas prácticas tecnológicas en la educación (asumiendo el uso dentro y fuera de las aulas) y ofreciendo interrogantes, a modo de reflexión pedagógica tales como

¿Cómo crear un espíritu crítico y dialogante si la posibilidad del diálogo real es cada vez más remota? ¿Cómo tener control sobre nuestra forma de trabajar cuando viene moldeada de antemano por los arquitectos del software? ¿Cómo no ver la forma en que las evaluaciones digitalizadas profundizan cada vez más la reducción de los alumnos a puros números, erosionan la posibilidad de una evaluación más holística e integrada? ¿Cómo incorporar factores emocionales y personales en herramientas que, por definición, despersonalizan y hacen abstractos a sus usuarios? (Gómez, 2020: 4)

\section{CONCLUSIONES}

La docencia ha sido pensada como vocación, trabajo, oficio y condición; categorías a partir de las que se ha intentado, desde diversos marcos teóricos y epistemológicos, dar cuenta de debates, contribuciones y obstáculos que atraviesan los procesos de construcción de subjetividades en las instituciones educativas. 
A partir de la búsqueda, la consulta y el análisis de la literatura socializada en este escenario actual que nos convoca, resulta ineludible interpelar la vinculación (y porque no pensar la promoción) de una burocratización acelerada de la labor docente. En este sentido, aspectos por el control de los cuerpos y de los desempeños profesionales se posicionan como cuestiones de tratamiento imprescindible intentando evitar procesos de uniformización del trabajo docente.

Así, la mayor parte de los aportes invitan no solo a no asumir acríticamente la virtualización del trabajo docente actual sino también a interpelar la digitalización de la educación como un nuevo destino.

Apelar a la construcción de sentidos a partir de la voz de los actores protagonistas impresiona ser el modo más adecuado de establecer reflexiones problematizadoras sobre el escenario que atraviesa la educación universitaria entendiendo que el trabajo docente se plantea como una categoría dinámica

Apelar a la construcción de sentidos a partir de la voz de los actores protagonistas impresiona ser el modo más adecuado de establecer reflexiones problematizadoras sobre el escenario que atraviesa la educación universitaria entendiendo que el trabajo docente se plantea como una categoría dinámica que es asumida, construyendo los docentes a lo largo de las distintas historias de vida posiciones en el trabajo cotidiano, permitiendo entender las condiciones de trabajo docente desde una definición que excede los recursos físicos, los tiempos y espacios institucionales (Rockwell y Mercado, 1990) apostando al carácter relacional de la tarea docente (Neufeld y Petrelli, 2017) potenciándose la incidencia y el valor de las relaciones con otros actores de la Universidad, convirtiéndose así en parte ineludible de las condiciones de trabajo. 


\section{BIBLIOGRAFIA}

Bonavida Foschiatti, C., y Gasparini, L. (2020). "El impacto asimétrico de la cuarentena". Documentos de Trabajo del CEDLAS

Esteban Bara, F. (2020). "COVID-19: la universidad debe renovarse en un entorno virtual sin perder su esencia". The Conversation, 2020.

Gómez, A. A. (2020). “Covid-19:¿Punto Sin Retorno de la Digitalización de la Educación?". Revista Internacional de Educación para la Justicia Social, 9 (3).

Villafuerte, J., Cevallos, Y. P., y Vidal, J. O. B. (2020). "Rol de los docentes ante la crisis del COVID-19. Una mirada desde el enfoque humano". REFCalE: Revista Electrónica Formación y Calidad Educativa. ISSN 1390-9010, 8 (1), 134-150

Rockwell, E. y Mercado, R. (1990). "La práctica docente y la formación de maestros". En E. Rockwell y R. Mercado (coord.), La escuela, lugar de trabajo docente (pp.63-78). México: DIE-CINVESTAV.

Neufeld, M. R. y Petrelli, L. (2017). "La experiencia escolar de niños pequeños en renovados contextos de desigualdad. Notas sobre la productividad de las representaciones sociales". En G. Novaro, L. Santillán, A. Padawer y L. Cerletti (coord.), Niñez, regulaciones estatales y procesos de identificación. Experiencias formativas en contextos de diversidad y desigualdad. (pp. 109132). Buenos Aires: Biblos. 